\title{
Canine renal cortical necrosis and haemorrhage following ingestion of an Amitraz-formulated insecticide dip
}

\author{
P A Oglesby ${ }^{\mathrm{a}}, \mathrm{K}$ E Joubert ${ }^{\mathrm{a}^{*}}$ and T Meiring ${ }^{\mathrm{b}}$
}

\begin{abstract}
Amitraz is a formamidine compound used in veterinary medicine as a topical dip to control ticks and mites on dogs and livestock. A 10-year-old female Scottish terrier was presented following the accidental oral administration of a dip containing amitraz. This case report describes the clinical signs, treatment and pathology of this dog. Clinical signs of toxicity from amitraz result from stimulation of alpha2-adrenergic receptors. Amitraz is seldom fatal because the effects can be reversed by alpha2-adrenergic antagonists. The dog recovered from the amitraz toxicity but died 5 days later from acute renal failure.
\end{abstract}

Key words: amitraz, acute renal failure, acute renal haemorrhage and necrosis, dog.

Oglesby P A, Joubert K E, Meiring T Canine renal cortical necrosis and haemorrhage following ingestion of an Amitraz-formulated insecticide dip. Journal of the South African Veterinary Association (2006) 77(3): 160-163 (En.). Fourways Veterinary Hospital, PO Box 68159, Bryanston, 2021 South Africa.

\section{INTRODUCTION}

Amitraz $\left(\mathrm{N}^{1}\right.$-[2,4-dimethylphenyl]$\mathrm{N}$-(2,4-dimethylphenyl)iminomethyl-Nmethylmethanimidamide) is a topical acaricide used in veterinary medicine for dogs and livestock ${ }^{7-9}$. The commercial preparation in the present case was Ectodex (Intervet SA, Isando), a formulation of $12.5 \% \mathrm{~m} / \mathrm{v}$ amitraz.

Amitraz toxicity has been described in the $\operatorname{dog}^{6,9,12}$. The most common form of amitraz toxicity in dogs is ingestion of amitraz-impregnated collars ${ }^{6,12}$. Amitraz toxicity is seldom fatal following ingestion if treated with an alpha antagonist $^{12}$. Most of the clinical signs of toxicity are related to amitraz's stimulation of alpha ${ }_{2}$ adrenergic receptors ${ }^{3,4,6,9,15}$. Dogs usually present with central nervous signs relating to depression and coma, respiratory depression, bradycardia, hypertension, mydriasis, gastrointestinal stasis, diarrhoea, vomiting, hypothermia, polyuria and hyperglycaemia ${ }^{6,13}$. Death from amitraz in animals is usually the result of respiratory failure from profound central nervous system depression ${ }^{14}$.

This case study describes acute renal failure in a dog following accidental oral administration of undiluted Ectodex formulation.

${ }^{a}$ Fourways Veterinary Hospital, PO Box 68159, Bryanston, 2021 South Africa.

'Section of Pathology, Department of Paraclinical Studies, University of Pretoria

${ }^{*}$ Author for correspondence.

E-mail: kenneth @ fourwaysvet.co.za

Received: September 2005. Accepted: July 2006

\section{CLINICAL HISTORY}

A 10-year-old, female Scottish terrier weighing $16 \mathrm{~kg}$ was presented with amitraz toxicity. Due to a misunderstanding at a pet boarding facility, the patient was orally dosed with an estimated $10 \mathrm{~m} \ell$ of amitraz (Ectodex, Intervet SA, Isando) instead of dipped. The patient was severely agitated with generalised muscle tremors, congested mucous membranes and miotic pupils. Tachycardia (198 beats per minute (bpm)), hyperventilation (168 breaths per minute (bpm)) and hyperthermia $\left(40.5^{\circ} \mathrm{C}\right)$ were evident. The presence of vomitus on the patient's tongue suggested she may have vomited before arrival at the veterinary hospital.

Thoracic auscultation and abdominal palpation were normal and examination of a peripheral blood smear revealed no blood parasites. Blood glucose and electrolytes were tested on admittance with an in-house blood chemistry analyser (Vet Test 8008, IDEXX Laboratories Inc, Maine, USA). Serum potassium was within normal range but the patient was mildly hyperglycaemic (Table 1).

The patient was sponged down with cold water and was given intravenous fluids (Ringers lactate, Fresenius Kabi, Midrand) at double maintenance rate $(80 \mathrm{~m} / \mathrm{h})$ and a bolus $(10 \mathrm{~m} / / \mathrm{kg})$ of hydroxyethyl starch (Voluven, Fresenius Kabi).

The mental state deteriorated rapidly. Within an hour the patient was comatose, menace and pupillary light reflexes were absent and the patient showed no response to stimuli except deep pain. At this stage the temperature had dropped to $35.1{ }^{\circ} \mathrm{C}$ and the mucous membranes had become mildly cyanotic. The patient was placed on a warm circulating water heating pad and wrapped in blankets. Oxygen was supplemented via a nasal cannula and a urinary catheter was inserted to monitor urine production. Urine specific gravity was measured with a refractometer and was greater than 1.040. Urine dipstick parameters were normal. The hypothermia took 8 hours to resolve.

As the risk of iatrogenic aspiration pneumonia was considered high, gastric lavage and treatment with activated charcoal was not carried out ${ }^{6}$. Anti-emetic therapy was started using metoclopramide (Clopamon, Fresenius Kabi) at $0.5 \mathrm{mg} / \mathrm{kg} \mathrm{i} / \mathrm{v}$ tid. Atipamizole (Antisedan, Novartis) was given at $0.06 \mathrm{mg} / \mathrm{kg} \mathrm{i} / \mathrm{m}$ every 5 hours as well as prophylactic antibiotics, cefazolin (Izaceph, Fresenius Kabi), at $20 \mathrm{mg} / \mathrm{kg}$ bid i/v.

Four hours after admission, heart rate (124 bpm) and respiratory rate (42 bpm) had improved. Systolic blood pressure (SBP), measured oscillametrically (Dinamap, Johnson \& Johnson, Midrand), was $129 \mathrm{~mm} \mathrm{Hg}$ and mean arterial pressure (MAP) was $95 \mathrm{~mm} \mathrm{Hg}$. Peripheral oxygen saturation was measured with a pulse oximeter placed on the tongue and remained above $95 \%$. Artificial tears (Teargel, Novartis, Isando) were applied to the corneas every 4 hours and the patient was turned every hour.

Three hours later, thoracic auscultation revealed bilateral pulmonary rales. Pulmonary oedema and acute respiratory distress syndrome (ARDS) were suspected. The fluid rate was dropped to $30 \mathrm{~m} / / \mathrm{h}$, a second bolus $(10 \mathrm{~m} / \mathrm{kg})$ of hydroxyethyl starch was administered and furosemide (Furosemide, Fresenius Kabi) at $2 \mathrm{mg} / \mathrm{kg}$ $\mathrm{s} / \mathrm{c}$ was given twice, 2 hours apart. Response to treatment was dramatic. The pulmonary oedema resolved, furosemide was discontinued and the fluid rate slowly increased over 13 hours to $80 \mathrm{~m} / \mathrm{h}$ (twice maintenance requirements). The higher fluid rates were necessary to maintain adequate blood pressure to preserve 
Table 1: Serum chemistry and electrolyte values.

\begin{tabular}{|c|c|c|c|c|c|}
\hline Parameter & Units & Day 1 & Day 2 & Day 4 & Reference values* \\
\hline \multicolumn{6}{|l|}{ Electrolytes } \\
\hline $\mathrm{Na}^{+}$ & $\mathrm{mmol} / \mathrm{l}$ & 163 & 166 & & $144-160$ \\
\hline $\mathrm{K}^{+}$ & $\mathrm{mmol} / \mathrm{l}$ & 4.3 & 3.8 & & $3.5-5.8$ \\
\hline $\mathrm{Cl}^{-}$ & $\mathrm{mmol} / \mathrm{e}$ & 117 & 131 & & $109-122$ \\
\hline \multicolumn{6}{|c|}{ Serum chemistry } \\
\hline Glucose & $\mathrm{mmol} / \mathrm{e}$ & 7.6 & 8.4 & 7.4 & $4.2-6.9$ \\
\hline ALT & $\mathrm{U} / \ell$ & & 95 & 17 & $8-80$ \\
\hline ALP & $\mathrm{U} / \mathrm{l}$ & & 1351 & 805 & $30-400$ \\
\hline Urea & $\mathrm{mmol} / \mathrm{l}$ & & 16.1 & 16.4 & $2.5-9.6$ \\
\hline Creatinine & $\mathrm{umol} / \mathrm{e}$ & & 241 & 205 & $44-159$ \\
\hline Total protein & $\mathrm{g} / l$ & & 64 & 57 & \\
\hline Albumin & $\mathrm{g} / l$ & & 24 & 18 & $27-38$ \\
\hline PCV & $\%$ & & 48 & & \\
\hline
\end{tabular}

*IDEXX Laboratories.

renal perfusion (mean arterial pressures above $60 \mathrm{~mm} \mathrm{Hg}$ and systolic pressures above $90 \mathrm{~mm} \mathrm{Hg}$ ). By carefully monitoring lung sounds and blood pressure and adjusting fluid rates accordingly, the MAP was maintained above $90 \mathrm{~mm} \mathrm{Hg}$ and urine output was $2.6 \mathrm{~m} / \mathrm{kg} / \mathrm{h}$.

On day 2 , the patient remained comatose and responsive only to deep pain. Atipamizole was continued every 5 hours. Owing to the presence of a fever throughout the day $\left(39.4-40.6^{\circ} \mathrm{C}\right)$, Metronidazole (Flagyl, Fresenius Kabi) at $20 \mathrm{mg} / \mathrm{kg}$ i/v bid was added to the existing antibiotic cover. Blood was taken for serum chemistry and electrolytes (Table 1). The patient had a haematocrit of $48 \%$, normal potassium levels and mild hyperglycaemia. Blood urea nitrogen and creatinine concentration and ALP activity were raised.

The elevated haematocrit suggested mild dehydration. However, perfusion seemed to be well maintained with intravenous fluids and colloids as the heart rate stayed between 108-140, the systolic blood pressure between 112-124 mm Hg, the diastolic blood pressure between 51-59 $\mathrm{mm} \mathrm{Hg}$ and the MAP at $75 \mathrm{~mm} \mathrm{Hg}$. Colloid administration was changed on the second day from bolus therapy to pulse therapy at $2 \mathrm{~m} / \mathrm{kg}$ every 2 hours.

Oxygen saturation was maintained above $95 \%$ without oxygen supplementation. Urine production was measured at an average of $2.0 \mathrm{~m} / \mathrm{kg} / \mathrm{h}$ over 24 hours. Furosemide ( $2 \mathrm{mg} / \mathrm{kg} \mathrm{s} / \mathrm{c}$ ) was given once to improve urine production. The patient vomited for the first time and developed a bloody diarrhoea. Metoclopramide was continued at $0.5 \mathrm{mg} / \mathrm{kg}$ s/c tid, but Cimetidine (Lenamet, Fresenius Kabi) at $10 \mathrm{mg} / \mathrm{kg} \mathrm{i} / \mathrm{v}$ tid, Sucralfate (Ulsanic, Aspen Pharmacare Ltd, Gallo Manor) at $10 \mathrm{~m} \ell \mathrm{p} / \mathrm{o}$ tid and Buprenorphine (Te mgesic, Schering Plough, Isando) were added to the therapy. A naso-oesophageal tube was inserted in the oesophagus to the level of the 7th intercostal space (confirmed by radiography) to facilitate nutritional support with an enteral feed (Survimed, Fresenius Kabi).

On day 3, the patient regained consciousness, showed interest in her environment, was ambulatory and could eat and drink on her own. Rectal temperature was normal throughout the day $\left(38.7^{\circ} \mathrm{C}\right)$. Tube feeding was changed to hand feeding every 4 hours. Fluids were continued but the rate was lowered to $38 \mathrm{~m} / \mathrm{h}$ and the colloids were discontinued. Medication continued unchanged except for the discontinuation of atipamizole.

The faeces were soft and brown without evidence of diarrhoea or fresh blood and there was no vomiting throughout the day. The patient pulled out her urinary catheter, which was not replaced owing to her clinical improvement and urine production was not measured. However, the patient was taken outside regularly and normal urination was observed 5 times throughout the 24-hour period. It was thought at this stage that the patient would make a full recovery so no blood samples were taken for serum chemistry analysis.

On day 4 , the patient vomited 3 times. In addition to metoclopramide, which had been part of the treatment protocol for 2 days, prochlorperazine (Stemetil, Aventis, Midrand) was added at $0.5 \mathrm{mg} /$ $\mathrm{kg} \mathrm{i} / \mathrm{m}$ tid and the patient was put back on naso-gastric tube feeding. The fluid rate was increased slightly $(50 \mathrm{~m} / \mathrm{h})$ to compensate for losses incurred by vomition. The antibiotics, analgesic and cytoprotective medications were continued. Serum chemistry (Table 1) revealed low albumin $(18 \mathrm{~g} / \mathrm{l})$, normal ALT but slightly raised ALP activity (805 U/l). Blood urea nitrogen and creatinine concentrations were elevated, $16.4 \mathrm{mmol} / \ell$ and $205 \mu \mathrm{mol} / \ell$ respectively, but were not significantly changed from previous days. The patient was still producing urine. An ultrasound examination of the abdomen showed a small amount of free abdominal fluid between the liver lobes. This was not aspirated. The echogenicity of the liver parenchyma appeared normal as did renal architecture.

The dog acutely decompensated and went into cardiac arrest on the morning of day 5. Ventricular fibrillation was diagnosed on an electrocardiogram. Cardiopulmonary resuscitation was immediately instituted. Blood taken during resuscitation for electrolyte determination revealed severe hyperkalaemia $(11.4 \mathrm{mmol} / \ell)$. The hyperkalaemia was considered the cause of the cardiac arrhythmia. Cardiopulmonary resuscitation was terminated after $10 \mathrm{~min}$ as the pupils had remained fixed and dilated and no return of spontaneous circulation had occurred. Permission was granted by the owner for a post mortem examination.

Macroscopic pathology revealed severe, ventral, subcutaneous oedema and moderate serosanguinous ascites. The left kidney was friable with severe peri-renal and diffuse renal haemorrhage. Half of the right kidney was similarly affected. Other abnormalities noted were severe diffuse hepatomegaly, friable adrenal glands, 2 gastric ulcers of $1 \mathrm{~cm}$ diameter each and severe pulmonary congestion in the caudo-dorsal lung lobes with multifocal areas of atelectasis.

Histopathological examination of the kidneys revealed severe, diffuse renal cortical haemorrhage, which resulted in obliteration of the interstitial tissue. Severe, acute, multifocal, glomerular and tubular necrosis was evident. The cells revealed increased eosinophilia, dissociation from the basement membrane, karyolysis and pycnotic nuclei. A few tubular epithelial cells contained golden yellow intracytoplasmic pigment (haemosiderin). About $50 \%$ of the glomeruli revealed a homoge- 
neous eosinophilic appearance with loss of cellular detail and few scattered nuclei of mesangial and endothelial cells still visible (glomerular necrosis). Moderate numbers of neutrophils infiltrated the interstitial tissue as a result of the necrosis. Mild, multifocal areas of fibrin deposition in the glomerular interstitium were evident with a few tubular lumens containing protein casts. Severe congestion of blood vessels was evident in the cortex with moderate congestion visible in the medulla.

The liver revealed severe, diffuse congestion with severe dilatation of the sinusoids with blood and resultant severe pressure atrophy of the hepatocytes. Mild, multifocal areas of fatty hepatosis and necrosis were also evident. The lungs showed moderate, diffuse congestion with severe accumulation of alveolar macrophages in the alveolar lumens. There was also a moderate fibrinous oedema in the lungs. Moderate congestion of brain blood vessels was observed. Histology of the thyroid gland, parathyroid gland, stomach, pancreas, gastro-intestinal tract and bone marrow were normal.

An incidental finding in this case was the presence of three $0.75 \mathrm{~cm}$ growths on the cranio-ventral urinary bladder mucosa, which were histologically diagnosed as papillary non-infiltrating transitional cell carcinomas.

\section{DISCUSSION}

The acute median oral lethal dose $\left(\mathrm{LD}_{50}\right)$ of amitraz in the rat is $800 \mathrm{mg} / \mathrm{kg}^{13}$. The $\mathrm{LD}_{50}$ for amitraz in dogs is $100 \mathrm{mg} / \mathrm{kg}$ while the toxic effect dose is $10 \mathrm{mg} / \mathrm{kg}^{6}$. This case study presents a dog that ingested an estimated $78 \mathrm{mg} / \mathrm{kg}$ of amitraz. Signs of amitraz toxicity usually appear 1 hour after oral administration and serum concentrations peak approximately 6 hours later ${ }^{12}$. This patient was dosed with amitraz more than 6 hours before arrival at the veterinary hospital. It was assumed, therefore, that the toxin had been fully absorbed.

Amitraz stimulates both alpha ${ }_{1}$ and alpha ${ }_{2}$-adrenoreceptors but most of the clinical effects observed are a result of its alpha $_{2}$-adrenergic agonistic activity ${ }^{4,10,13}$. In the rat, amitraz is a potent inhibitor of monoamine oxidase and prostaglandin $\mathrm{E}_{2}$ synthesis but the role of this in amitraz poisoning in dogs is unknown ${ }^{14}$. The alpha $a_{2}$-adrenoreceptor effect produces central nervous system and respiratory depression, bradycardia, gastrointestinal stasis, diarrhoea, vomiting and hypothermia'. Hypotension is seen in humans and guinea pigs but peripheral vasoconstriction results in hypertension in $\operatorname{dogs}^{2,3,10,14}$. Hypertension and vasoconstriction is followed by a decrease in heart rate and cardiac output ${ }^{2}$. The decrease in heart rate and cardiac output are not solely the result of a baroreceptor reflex from hypertension because amitraz inhibits the baroreceptor reflex and yohimbine restores $i^{16}$. Reduced baroreceptor function could lead to cardiovascular collapse ${ }^{16}$. This was seen in our patient, which required intravascular volume support to maintain blood pressure.

As an antidote, atropine is not recommended to counteract bradycardia caused by amitraz toxicity because it does not reverse all alpha $a_{2}$-agonist effects on the cardiovascular system $^{9-10}$. While increasing heart rate it may potentiate hypertension'. The resulting increase in myocardial oxygen consumption may predispose to cardiac arrhythmias. Atropine also aggravates gastrointestinal hypomotility

Amitraz effects on ventilation include a decrease in respiratory rate, tidal volume and minute ventilation ${ }^{2}$. The hypoxaemia in this patient was most likely the result of pulmonary oedema. Respiratory rate remained high and therefore it seems unlikely that amitraz caused respiratory depression. Miosis occurs in man but mydriasis dominates in $\operatorname{dogs}^{14}$. Besides species differences, clinical signs are also dose-dependent. Bradycardia and respiratory depression are replaced by tachycardia and hyperventilation in severe intoxication ${ }^{2}$. Bradycardia was not noted in this patient. This was either a result of the high dose of amitraz administered or a consequence of systemic shock (hypotension, acidosis) and circulatory compromise.

$\mathrm{Alpha}_{2}$-adrenergic stimulation inhibits insulin release from the pancreas and increases glucagon which causes hyperglycaemia $^{5,13,17}$. A mild persistent hyperglycaemia was seen in this case. Hyperglycaemia has been reported in humans exposed to amitraz and following experimental administration of amitraz $z^{5,12,14}$. Topical application and intravenous administration of amitraz has been shown to increase baseline plasma glucose levels and blunt insulin response following a glucose challenge $\mathrm{e}^{11,17}$. Caution should therefore be exercised when amitraz is used in diabetic dogs. Yohimbine can reverse the effects of amitraz on insulin ${ }^{17}$. Insulin levels increase rapidly and glucose decreases after administration of an alpha $a_{2}$-antagonist and this can be used to assist in the diagnosis of amitraz toxicity ${ }^{12}$. Amitraz also decreases antidiuretic hormone $(\mathrm{ADH})$ and renin secretion, which leads to an increase in urine production ${ }^{5}$. In human patients, an increase in urine production has been noted following intoxication ${ }^{5}$.
Yohimbine is an alpha ${ }_{2}$-adrenergic antagonist and will competitively displace amitraz from the alpha 2 -receptors thus reversing central nervous system depression, bradycardia, hypertension and gastrointestinal effects ${ }^{10}$. It may cause tachycardia and tachypnoea. Atipamizole, another specific alpha ${ }_{2}$-adrenergic antagonist, may be superior to yohimbine as an amitraz antagonist because it has less cardiorespiratory effects. This patient was given 6 treatments of atipamizole at $0.06 \mathrm{mg} / \mathrm{kg} \mathrm{i} / \mathrm{m}$ at $5-\mathrm{h}$ intervals. A dose of $0.05-0,2 \mathrm{mg} / \mathrm{kg} \mathrm{i} / \mathrm{m}$ is recommended although the lower dose is usually sufficient $^{12}$. As the half-life of atipamizole is short ( $<2$ hours) and the elimination half-life of amitraz dose dependant (1.5-2 hours for doses of $1 \mathrm{mg} / \mathrm{kg}$ and 23.6 hours for doses of $100 \mathrm{mg} / \mathrm{kg}$ ), atipamizole may be repeated at 2- to 4-hour intervals if necessary to control symptoms ${ }^{6,12}$.

Amitraz produces neurological depres$\operatorname{sion}^{12}$. The neurological depression in this patient lasted 2 days. This would support the longer half-life of amitraz following higher doses.

Amitraz is metabolised in the liver and will cause dose-dependant hydropic and fatty changes in hepatocytes ${ }^{8}$. Hepatic injury was evident at necropsy in this patient.

Acute renal failure in this patient was most likely the result of both toxic and anoxic insults following poisoning. At presentation marked shock was evident, which would cause renal hypoxia/anoxia. The patient responded positively to therapy, showing adequate urine production, normal serum $\mathrm{K}^{+}$, and stable urea and creatinine concentrations. This led to an erroneous clinical underestimation of the renal damage. It is interesting to note that clinical improvement occurred despite more than $75 \%$ of the renal cortex having been injured or necrotic. Ultimately the patient died due to the inability of the kidneys to excrete potassium.

In a retrospective case study of hospital acquired renal failure, histopathology was the only definitive evidence of acute renal failure in 5 of $29 \mathrm{dogs}^{1}$. In another 5 cases, acute renal failure, diagnosed on serum urea and creatinine concentrations, appeared to resolve based on serum chemistry yet acute tubular injury was still present on histopathology ${ }^{1}$. The above 2 scenarios indicate the difficulties in diagnosing acute renal failure by serum chemistry in some individuals. This case mimicked the second scenario whereby a clinical diagnosis of renal injury was made but the extent of this damage was underestimated due to clinical improvement of the patient after therapy. The 
authors therefore suggest intensive monitoring of renal function in cases of amitraz toxicity. In addition to continued monitoring of urine production and BUN and creatinine concentrations, urine sediment should be examined daily for casts and urine solute excretion measured.

\section{REFERENCES}

1. Behrend E N, Grauer G F, Mani I, Groman R P, Salman M D, Greco D S 1996 Hospital-acquired acute renal failure in dogs: 29 cases (1983-1992). Journal of the American Veterinary Medical Association 208: 537-541

2. Cullen LK, Reynoldson J A 1987 Cardiovascular and respiratory effects of the acaricide amitraz. Journal of Veterinary Pharmacology and Therapeutics 10: 134-143

3. Cullen L K, Reynoldson J A 1990 Central and peripheral $\alpha$-adrenoreceptor actions of amitraz in the dog. Journal of Veterinary Pharmacology and Therapeutics 13: 86-92

4. Cullen L K, Reynoldson J A 1990 Effects of amitraz on nerve conduction and neuromuscular transmission in anaesthetised dogs. Research in Veterinary Science 48: 162-164
5. Doganay Z, Aygun D, Altintop L, Guven H, Bildik, F 2002 Basic toxicological approach has been effective in two poisoned patients with amitraz ingestion: case reports. $\mathrm{Hu}$ man and Experimental Toxicology 21: 55-57

6. Duncan K L 1993 Treatment of amitraz toxicosis. Journal of the American Veterinary Medical Association 203: 1115-1116

7. Estrada-Pena A, Rème C 2005 Efficacy of a collar impregnated with amitraz and pyriproxyfen for prevention of experimental tick infestations by Rhipicphalus sanguineus, Ixodes ricinus, and Ixodes scapularis in dogs. Journal of the American Veterinary Medical Association 226: 221-224.

8. Filazi A, Gûvenç T, Kum C, Sekkin S 2004 Pathological findings in acute amitraz intoxication in mice. Turkish Journal of Veterinary Animal Science 28: 873-878

9. Grossman M R, Garvey M S, Murphy M J 1993 Amitraz toxicosis associated with ingestion of an acaricide collar in a dog. Journal of the American Veterinary Medical Association 203: 55-57

10. Hsu W H, Lu Z-X 1986 Effects of amitraz on heart rate and aortic blood pressure in conscious dogs: influence of atropine, prazosin, tolazoline, and yohimbine. Toxicology and Applied Pharmacology 84: 418-422
11. Hsu W H, Schaffer D D 1988 Effects of topical application of amitraz on plasma glucose and insulin concentrations in dogs. American Journal of Veterinary Research 49: 130-131

12. Hugnet $C$, Buronfosse F, Pineau $X$, Cadoré J-L, Lorgue G, Berny P J 1996 Toxicity and kinetics of amitraz in dogs. American Journal of Veterinary Research 57: 1506-1510

13. Jones R D 1990 Xylene/Amitraz: a pharmacologic review and profile. Veterinary and Human Toxicology 32: 446-448

14. Leung V K S, Chan T Y K, Yeung V T F 1999 Amitraz poisoning in humans. Clinical Toxicology 37: 513-514

15. Queiroz-Neto A, Zamur G, Goncalves S C, Carregaro A B, Mataqueiro MI, Harkins JD, Tobin T 1998 Characterization of the antinociceptive and sedative effects of amitraz in horses. Journal of Veterinary Pharmacology and Therapeutics 21: 400-405

16. Reynoldson J A, Cullen L K 1996 Amitraz depresses cardiovascular responses to bilateral carotid occlusion. Journal of Veterinary Pharmacology and Therapeutics 19: 22-26

17. Smith B E, Hsu W H, Yang P-C 1990 Amitraz-induced glucose intolerance in rats: antagonism by yohimbine but not by prazosin. Archives of Toxicology 64: 680-683 